\title{
Convivencia escolar: una aproximación para la adquisición de conocimientos mediante la investigación como estrategia pedagógica ${ }^{1}$ \\ School life: an approach based on research as a pedagogical strategy
}

DOI: http://dx.doi.org/10.17981/cultedusoc.9.3.2018.24

Artículo de investigación. Fecha de recepción: 15/06/2018. Fecha de aceptación: 27/11/2018

Elizabeth Pertuz Rodríguez"

Evelia Roncollo Móscate; Ana Martínez Vergara; Marlon Santofimio Cortina; Efraín Henao Castillo; Surith Buendía Yépez y José Teherán Velásquez3

Institución Educativa Departamental Gabriel Escobar Ballestas, Sede Hugo Acero Cáceres (Colombia) pochola2006@gmail.com

Para citar este artículo:

Pertuz, E., Roncollo, E., Martínez, A., Santofimio, M., Henao, E., Buendía, S. y Teherán, J. (2018). Convivencia escolar: una aproximación para la adquisición de conocimientos mediante la investigación como estrategia pedagógica. Cultura. Educación y Sociedad 9(3), 209-218. DOI: http://dx.doi.org/10.17981/cultedusoc.9.3.2018.24

\section{Resumen}

La educación en Colombia está generando acciones para que las instituciones educativas municipales promuevan una formación en competencias ciudadanas que permita fortalecer la convivencia escolar, por tal razón se plantea la presente investigación, que tuvo como objetivo comprender la importancia de la convivencia escolar en la adquisición de conocimientos mediante la investigación como estrategia pedagógica (IEP). Esta investigación se encuentra orientada bajo un enfoque cualitativo, con abordaje desde la investigación como estrategia pedagógica; la unidad de análisis estuvo conformada por 20 estudiantes entre las edades de 7 a 13 años de los grados segundo tercero y cuarto de la Escuela Urbana Mixta Hugo Acero, del Municipio de Plato, Magdalena. Como resultado se implementaron espacios recreativos para la sana convivencia escolar desde el área de ética y valores, apoyada por asignaturas como lengua castellana, religión, orientado desde los resultados de las investigaciones de los estudiantes frente la problemática de convivencia, logrado mediante la incorporación de la Investigación como estrategia pedagógica al currículo, permitiendo la implementación del proyecto de convivencia en el aula, implementando los conocimientos en el área central y complementaria y los conocimientos construidos en la dinámica de las relaciones sociales.

Palabras clave: convivencia escolar, investigación como estrategia pedagógica, escuela.

\section{Abstract}

Education in Colombia is generating actions so that the municipal educational institutions promote a training in civic competences that allows strengthening school coexistence; Given this, the present investigation aimed to analyze school coexistence as an approach from research as a pedagogical strategy (IEP) oriented with a qualitative approach, with an approach based on research as a pedagogical strategy; The analysis unit consisted of 20 students between the ages of 7 and 13 years of the second and fourth grades of the Urban Mixed School Hugo Acero, of the Municipality of Plato, Magdalena. As a result, recreational spaces for healthy school coexistence were implemented from the area of ethics and values, oriented from the results of students' research on the problem of coexistence, achieved through the incorporation of Research as a pedagogical strategy into the curriculum, allowing the implementation of the project of coexistence in the classroom, implementing the knowledge in the central and complementary area and the knowledge built in the dynamics of social relations.

Keywords: school coexistence, research as a pedagogical strategy, school.

1 Este artículo ha sido derivado del Programa de Fortalecimiento de la Cultura Ciudadana y Democrática CT+I a través de la IEP apoyada en TIC en el Departamento de Magdalena: CICLON

2 Líder del Grupo de Investigación "El Ciclón Hugocacerista".

3 Docentes de la Institución Educativa Departamental Gabriel Escobar Ballestas, Sede Hugo Acero Cáceres y miembros del Grupo de Investigación: El Ciclón Hugocacerista.

- The author; licensee Universidad de la Costa - CUC.

Cultura, Educación y Sociedad vol. 9 no. 3, pp. 209-218. Diciembre, 2018

Barranquilla. ISSN 2389-7724 Online 


\section{Introducción}

La convivencia escolar, hace referencia a las relaciones interpersonales tolerantes y respetuosas que se generan en la comunidad educativa, lo cual fomenta un clima escolar adecuado para el desarrollo de los procesos educativos, enmarcados en la integridad y la humanización (García y Niño, 2018).

No obstante, ese concepto de convivencia idealizado no se observa en las instituciones educativas, por la existencia de problemas de violencia y exclusión en los niños, niñas y adolescentes, estos pueden estar asociados a antecedentes de desarrollo inadecuado de tareas y habilidades básicas, lo que alerta de inmediato a detectar esas habilidades mal aprendidas para proporcionar experiencias que ayuden a su adquisición (Díaz, 2002).

La convivencia escolar es un concepto estudiado desde distintas ópticas en al ámbito académico, debido a la complejidad de los crecientes problemas sociales de violencia e inseguridad que se enfrenta a nivel mundial en las instituciones educativas, lo cual requiere que se continúen generando acciones efectivas adecuadas a las necesidades de los actores que conforman la escuela (Arias, 2018).

Sin embargo, los modelos tradicionales que sostienen a las instituciones son aspectos relacionados con la jerárquica, rigidez y autoritarismo, limitando la posibilidad de empoderamiento, participación de estudiantes y docentes, excluyéndoles de las decisiones de la comunidad educativa, a pesar de existir distintos grupos y representantes de los mismos, quitando sentido de pertenencia por ende poca apropiación de la norma; Asimismo, influye con la adquisición de los conocimientos, legitimado la idea de estudiantes ejecutores de órdenes que su única función es repetir discursos y cumplir normas, estas ideas tradicionalistas no permiten el desarrollo de habilidades como el pensamiento crítico. De igual forma, afecta a los docentes quitando autonomía en sus estrategias pedagógicas innovadoras que dinamicen el proceso enseñanza-aprendizaje (De la Concepción, 2015).

Algunos autores como Perales et al., (2014) y Arrieta (2016) plantean que, la democracia diversa e inclusiva, dentro de la toma de decisiones en las instituciones generan mayor convivencia escolar. Promoviendo una cultura basada en la paz y la tolerancia, enseñando de esta manera a incluir a otros en procesos sociales y afectivos, reforzando distintos valores en la comunidad estudiantil, que logran verse reflejados en el aula de clase.

En este entendido, exponen Hernández y Jiménez (2015), el reclamo no solo es de "ética individual de las personas que componen las organizaciones, sino que ven un ente con responsabilidad propia... a la que debe exigírsele comportamientos acordes a la misma" (p. 11), según su razón de ser.

Así entonces, uno de los principales propósitos de las instituciones educativas es lograr una buena convivencia entre toda su comunidad educativa, debido a la importancia que tiene en el proceso de aprendizaje, y así como a la contribución en las relaciones interpersonales, que proporciona un clima adecuado en el aula de clases (López, Carvajal, Soto y Urrea, 2013).

Si bien es cierto que, en las instituciones educativas, debería existir un buen clima educativo, en distintas ocasiones no se da de esta manera, por el contrario se han desarrollado situaciones poco agradables, que no contribuyen con el aprovechamiento del proceso de enseñanza-aprendizaje (García, Cantó, López, Miñarro, Martinez,2011). Lo que conlleva a buscar estrategias que permitan implementar nuevas medidas que nutran dicho proceso. 
En la actualidad, algunos espacios públicos no se utilizan en lo absoluto para ningún tipo de actividad, otros ya se han fortalecido con actividades que tienen precisamente una relación directa con el espacio, puesto que les dan mucha más importancia y fuerza a aspectos como a recreación, la economía y turismo al tiempo que recrean y reunen a la comunidad (Ocampo, 2008).

La investigación como estrategia pedagógica es vista como una herramienta educativa que permite generar visiones innovadoras en el proceso de enseñanzaaprendizaje desde una perspectiva crítica, puesto que crea escenarios de saber escolar que se convierten en la posibilidad de transformar las relaciones entre los miembros de una comunidad, y con ello emplear metodologías de enseñanza basadas en el aprendizaje contextual, generando practicas pedagógicas que permiten la exploración, indagación y análisis de la información, que permite contrastar la teoría con la práctica (Mejía y Manjarrés, 2010).

En este entendido, Arellano (2004) citado por Mendoza \& López (2015), expresa que la investigación como estrategia debe ser concebida similar al pensamiento estratégico que según "hace referencia a la forma del pensamiento que se dirige hacia la creatividad, imaginación, y búsqueda de opciones innovadoras y realistas, como potenciación de lo posible enmarcado dentro de un proyecto de construcción intencional de la realidad". (p. 154). Las escuelas no escapan de ello y es de allí que, se hace preciso introducir una gestión estratégica como una preocupación social (Mendoza, López y Salas, 2016)

Por lo anterior se toma a la investigación como estrategia pedagógica IEP como modelo para el desarrollo del proyecto investigativo, de igual manera se presentan otros intereses lúdicos desarrollados por los niños, observados en la práctica docente, surgiendo motivación de abordar el juego como estrategia para favorecer el aprendizaje, de cierta formara ayudara a los docentes a acercarse con mayor facilidad a los estudiantes (Romero, 2015).

Desde los primeros años los niños comienzan a desarrollar habilidades para participar en procesos de comunicación e indagación, por eso desde muy pequeños se deben acostumbrar a estos a ambientes alegres, lúdicos y donde haya más niños con los que puedan relacionarse, nuevos conocimientos e incógnitas frente al funcionamiento del entorno que le rodea, relacionándose y acostumbrándose para cuando entren a un espacio de mayor dificultad (Meléndez, Juárez, 2012).

Medina y Quintero (2003), mencionaron que la recreación es una estrategia para la mejora de la convivencia, considerando la vital importancia que tiene el juego durante los primeros años de vida de los individuos, y después de ponerse a pensar un poco sobre el gran valor que se le da a este como estrategia de enseñanza y aprendizaje, se desarrollan estudios que abarquen el juego como estrategia didáctica para enriquecer el lenguaje de los niños.

A través de actividades motrices, y ejercicios de expresión corporal para niños, se establecen estrategias que permitan a estos desarrollar habilidades creativas, adquiriendo la integración, la convivencia, la comunicación y la estimulación de las habilidades para expresar los sentimientos (Guamani, 2015).

Cuando se escucha de casos de bullying, muchos maestros prefieren creer que se debe a casos aislados y que su escuela, aún más, su grupo está exento de dicho problema, pues a veces es difícil entender como alumnos que se han formado bajo una "buena educación", tienden a ser estudiantes conflictivos. Lo que lleva a que descuide y no se haga un seguimiento adecuado a estos estudiantes, lo que posteriormente tiene a desarrollar estudiantes problema (Manilla, 2014). 
A lo largo del tiempo se han analizado la forma de enseñanza de los niños, teniendo en cuenta que, si esta no se hace en un lugar adecuado, los niños pueden crecer con cierto tipo de ideas, que quizá no sean de mucha ayuda durante su crecimiento, de esto surge la idea de crear ambientes sanos, divertidos y de recreación, para que los niños se sientan cómodos y puedan desarrollar sus habilidades de una forma más precisa (Rivera, 2012).

El concepto de educación hace referencia a ir más allá de aspectos rigurosamente académicos. La educación hace también referencia a la formación integral de los seres humanos, basada en valores universales que permitan desarrollar una mejor convivencia social. La idea de formar buenos estudiantes, no se basa en formar niños pasivos y obedientes, sino que al momento de jugar e interactuar con los demás compañeros, lo hagan de una forma sana, sin utilizar palabras inadecuadas o violentas, la idea es que sean más activos y creativos dentro de sus posibilidades (Mejía, 2017; García, 2003).

De igual forma, la educación integral, busca de manera directa un aprendizaje buscando fortalecer la personalidad de los estudiantes, de manera ética, critica, creativa, permitiendo ser participe y solidario, con capacidades para proponer e interactuar en su entorno, construyendo identidad cultural (Lugo, 2007).

Dentro de la sociedad, la educación es la piedra angular que resuelve muchos problemas sociales, e incluso puede llegar a transformar a una persona y el entorno en el que esta se encuentra. La ausencia de reconocimiento y respeto por el otro, es por ello que se hace énfasis en indagar y actuar sobre las diferentes estrategias diseñadas al interior de las escuelas con el fin de dinamizar los procesos de enseñanza, buscando motivar y despertar el interés del niño por aprender, por interactuar en su entorno y por desarrollar habilidades y comportamientos en pro de la convivencia escolar.

La falta de espacios de recreación, ha generado consecuencias a nivel de resultados académicos y afecta directamente la convivencia, de manera que los estudiantes muestran conductas agresivas, utilización de palabras soeces, irrespeto por el otro y por la institución. Estos problemas de convivencia se encuentran directamente relacionados con el contexto familiar y social en el que viven los estudiantes, así mismo la falta de espacios para la socialización y la aprehensión de buenos hábitos en la escuela.

Se plantea que existe una estrecha relación entre la convivencia escolar y los procesos de aprendizaje (Herrera y Rico, 2014). Es decir, los establecimientos educativos donde se mantienen mejores relaciones entre educandos, docentes, familias y el grupo de directivos docentes, es decir, donde existe un mejor clima escolar, el grupo de educandos se siente involucrado en mejores procesos de enseñanza y obtiene mejores resultados académicos.

En ese sentido, considerar la investigación como estrategia pedagógica implica recrear un espacio de inclusión, donde se respete y se viva la diversidad haciendo evidentes los canales de la creatividad del docente, para convertir las preguntas de los grupos de aprendizaje colaborativo conformados por niños, niñas y adolescentes en una investigación, llevándolos a tomar un rol activo en el proceso de aprendizaje mediante la construcción de su propia conocimiento, potenciando así, las habilidades y destrezas de los estudiantes, convirtiendo la educación en un vehículo de capacitación, de oportunidades y de recreación (Camargo, 2015).

Desde la metodología propuesta por la IEP los contenidos curriculares se convierten en problemas, donde el docente a partir de las preguntas planteadas por los estudiantes diseña unas trayectorias de 
indagación, que orientan todo su proceso de investigación y generación de saber y conocimiento (Pozo \& Gómez, 2006).

De lo mencionado anteriormente, surge la estrategia de los patios como el espacio propicio para poner al servicio de las necesidades infantiles, diversas actividades que les permitan opinar libremente con respeto, moverse, explorar, jugar, querer, con capacidades para adquirir normas y valores. Características propias de su edad que se buscan potencializar a través del juego y la lúdica. Por lo tanto, no se únicamente de la infraestructura o de un plano físico, si no, de un espacio que permita interactuar con el medio ambiente, su entorno social y su cultura (Parra, 2005).

Estos espacios surgen por la necesidad de crear ambientes que impacten directamente en la conducta de los estudiantes y en la calidad de la enseñanza, buscando mitigar condiciones que dificultan el desarrollo y aprendizaje de los niños y las niñas, teniendo en cuenta que cad espacio de interacción se configura como un escenario educativo (Rodríguez, 2018).

Asimismo, por la necesidad de fortalecer las interacciones sociales que se presentan en la institución, en aras de mejorar la convivencia escolar, donde prima el proceso investigativo como el elemento mediador entre el proceso de aprendizaje y la convivencia escolar, como elementos articulados para el desarrollo de capacidades de creación e innovación de los maestros y estudiantes, que se empiece a despertar el interés por la ciencia tanto en el cuerpo profesorado como en el alumnado, con la convicción de asumir un nuevo rol social y dinamizar el quehacer pedagógico y educativo desde la investigación, pues es esta la fuente y el pilar que concibe y hace al maestro un actor de cambio e instrumento innovador y de calidad para cumplir con las necesidades y los cambios que se presentan en la sociedad actual.
La propuesta metodológica se fundamenta en valores que buscan desarrollar capacidades de cognitivas, afectivas, valorativas y de acción. Asumir la investigación desde modelos educativos críticos y estrictos, supone establecer una lucha teórico-prática en la que se definen aspectos como el conocimiento, la cultura, lo humano y los grupos sociales de manera objetiva, que ha contribuido a la generación de formas de poder que han conducido al control de las desigualdades en la sociedad (Ortega, Peñuela y López, 2009).

En resumen, el docente desde la IEP parte de la pregunta de sentido común de los estudiantes, la redirecciona a la perspectiva de la educación, reconociendo la existencia de saberes comunes o elaborados, asociando los conocimientos disciplinares con las experiencias socioculturales, promoviendo así la apropiación practica desde el pensamiento crítico buscando transformar contextos, culturas, epistemologías, y redirigir sus escenarios de poder para construir subjetividades y ciudadanías (Mariño, 2010).

\section{Metodología}

\section{Diseño}

El estudio estuvo orientado a partir de un enfoque cualitativo, la metodología implementada en el proyecto de los Patios es el de la Investigación como estrategia Pedagógica (IEP), la propuesta se encuentra basada en la relación existente entre los saberes académicos y los saberes de la cultura, evidenciados en la sociedad; Por ello, la investigación desarrollada busca a través de una propuesta metodológica realizar el reconocimiento social de los actores, enlazando la educación que brinda la institución y la sociedad, realizando una labor integral en los planteles estudiantiles, debido a que son los estudiantes guiados por los docentes quienes ponen en 
juego a través de la negociación cultural de sus preguntas y diferentes concepciones los distintos planteamientos problemas que piensan investigar, emprendiendo las decisiones de su trayecto y las herramientas a utilizar. (Mejía y Manjarrez, 2010). Como lo son las TIC'S, quienes permiten dentro de la investigación no solo conocer conceptos si no sustentar las ideas desde la academia observadas en la sociedad, cumpliendo con la relación anteriormente mencionada.

\section{Participantes}

La unidad de análisis estuvo conformada por veinte (20) estudiantes entre las edades de 7 a 13 años integrantes de los grados de segundo, tercero y cuarto grado, de la Escuela Urbana Mixta Hugo Acero, del Municipio de Plato, Magdalena.

\section{Técnicas e instrumentos}

Para el presente estudio se seleccionaron como técnicas de recolección de datos la observación y la bitácora del investigador. La observación, permite registrar cada aspecto del proceso a estudiar en la bitácora, lo cual permite, percibir e identificar la realidad tal cual como la viven los actores.

Este proceso se llevó a cabo inicialmente en los descansos de los estudiantes, pues son los momentos y espacios donde más se presentan situaciones conflictivas en la institución, analizando aspectos como; actitudes y formas de comunicación con los demás compañeros y docentes, trabajo y juegos en equipo, respuestas ante situaciones incomodas y la tolerancia frente a actitudes de los demás. Seguidamente, se realizó el proceso dentro del aula, en el desarrollo de cada clase, observando y describiendo cada uno de los anteriores aspectos, debido a su importancia, marcando una trayectoria para continuar en el proceso dependiendo de la respuesta obtenida en la observación y el grupo focal conformado por estudiantes se decido si era pertinente la intervención. Lo primero que se llevó a cabo fue una observación directa en las actividades realizadas en el patio y en los salones de la institución, donde se registraron en un cuaderno de notas los comportamientos, conductas, las normas y reglas en los juegos y actitudes de los niños, la cooperación y el trabajo en equipo en las actividades que realizaban.

\section{Procedimiento}

Los participantes fueron seleccionados intencionalmente porque se encontraban relacionados directamente con la problemática observada, se seleccionó un espacio diferente para trabajar desde el área de ciencias sociales, Inicialmente se llevó a cabo un proceso diagnóstico de observación por parte de los docentes para identificar las conductas que debilitaban la convivencia escolar seguidamente se desarrolló la investigación teniendo en cuenta los pasos planteados en la ruta metodológica de la IEP, estableciéndose seis etapas, la primera fue la creación y distribución de grupos de aprendizaje colaborativo con los participantes del estudio, donde se mostró toda la problemática, segundo se les solicito construir preguntas problematizadoras sobre la convivencia escolar, tercero, se hicieron revisiones bibliográficas y se plantearon soluciones hipotéticas a dicho problema, identificando causas y efectos a nivel teórico, por medio del apoyo de las TIC'S, donde a partir de videos en YouTube y documentales lograron observar los problemas de convivencia presentados en diferentes lugares y las estrategias que habían utilizado para darle solución a los mismos. Cuarto se diseñaron los espacios dentro de las aulas de clases y el patio donde se realizaría el desarrollo de la investigación guiadas por el docente En el quinto paso se recolecto 
información de los trabajadores y docentes externos al grupo perteneciente a la investigación a través de una negociación cultural y de esta manera contrastar los distintos conocimientos y la teoría investigada. Realizando una síntesis de los resultados, como sexta etapa; Asimismo, la reflexión de la práctica y la apropiación del plantel estudiantil, a través de una feria evidenciando la significancia de la experiencia, donde los estudiantes presentaron sus propuestas, estas estaban orientadas hacia el uso de la democracia, donde todos los niños, niñas y jóvenes de la institución pudieran alzar su voz para tomar decisiones donde ellos se encontraran involucrados, donde a través de la participación activa, pudieran generar diferentes formas de tramitar los conflictos, siendo más tolerantes con la diferencia, finalmente los estudiantes sistematizaron la información mediante cuentos, historietas, poemas y canciones.

\section{Resultados}

Partiendo de los resultados obtenidos desde el primer ejercicio de observación en los descansos, los docentes lograron identificar que la mayor dificultad de los estudiantes era la forma de tramitar o solucionar los conflictos, donde muchas veces la falta de tolerancia, traspasaba la esfera oral y concluía en agresiones físicas, sin embargo, en medio del proceso, se observó que las sanciones realizadas por la escuela no eran suficientes puesto que los problemas continuaban al salir de la institución, en ese sentido, se realizaron grupos de aprendizaje colaborativo y se expusieron las acciones observadas que generaban un clima escolar desfavorable, ante estas situaciones, los estudiantes generaron diversas preguntas problematizadoras y se les oriento hacia la búsqueda de información utilizando como herramienta las tabletas, indagando en distintos canales para la observación de la problemática.
Para hacer la reflexión y sistematización de la práctica a la comunidad estudiantil se realizó una feria estudiantil, evidenciando estos videos y documentales, relatando historias y resaltando la importancia de la democracia a partir de las ciencias sociales y éticas y valores. Incentivando a los estudiantes a dar su punto de vista de las cosas dentro del respeto y la tolerancia aportando a la democracia de la institución aportando sentido de partencia en cada una de sus intervenciones dentro y fuera del aula de clases, aumentando de esta manera el hábito de lectura y las habilidades investigativas para hacer un aporte significante.

Por lo cual se crearon comités estudiantiles, a los cuales se debían acercar los estudiantes que tuvieran diferencias, exponer sus casos y entre toda la comunidad buscar un una solución donde ambas partes se encontraran beneficiadas y se diera una tramitación adecuada del conflicto mediante la conciliación, entregando así herramientas que permiten la consolidación de competencias ciudadanas, para resolver la problemática de convivencia observada en la institución partiendo de la integración de la investigación como estrategia pedagógica.

\section{Discusión}

Esta investigacion basadas en el método de la investigacion como estrategia pedagógica, permite obtener resultados como los anteriormente expuestos, permitiendo observar la necesidad de crear espacios de participación estudiantil, donde los niños, niñas y adolescentes puedan expresar sus necesidades y sentimientos, a través de un docente guía, potencializando habilidades investigativas, donde obtengan bagaje teorico para proponer y decidir, conociendo sus derechos y de igual forma, escuchando sus opiniones y pensamientos, tanto de ellos como lo de sus compañero, comprendiendo la importancia de los valores y como estos constituyen sociedad, evidenciado en la historia, en áreas como ciencias sociales, sino, en la 
comunidad donde se desarrollan de manera integral a diario (Mejía, 2011), entendiendo que la solución no está en el autoritarismo, sino en mostrar la amplitud de un problema, desglosarlo, revisar sus implicaciones, crear conciencia sobre los efectos que puede tener un comportamiento, donde el propio estudiante sea capaz de tener una mirada crítica frente a un problema y proponer soluciones al mismo.

Asimismo, a la luz de la creación de espacios de participación, estos resultados se sustentan con los planteamientos de autores como: Perales, Arias \& Bazdresch, (2014) quienes expresan que, para generar una sana convivencia en las instituciones educativas, es necesario orientar prácticas democráticas, que permitan aceptar la diversidad, la inclusión y una formación en cultura de paz que sea de carácter transversal, lo cual no se encuentre enfocado en el aprender a vivir con otros, sino involucrar otros procesos como los núcleos socio-afectivos de confianza, respeto, cuidado y responsabilidad, asociado a las acciones y actuaciones que se observan en el contexto escolar cotidianamente.

A manera de conclusión se observa que la convivencia en las instituciones no es fácil, y siempre existirán choques de ideas, pero la diferencia radica en la tramitación de los conflictos, donde los resultados pasarían de ser un problema, a una simple experiencia de la que se debe aprender. Cada uno de los sitios y espacios que la institución brinde a un estudiante deberán ser de suma comodidad, de manera que el estudiante se sienta familiarizado y no le sea difícil desarrollar estrategias de convivencia, basadas en la participación a su vez los docentes deben continuar con el acompañamiento de los alumnos, para poder intervenir en cualquier situación que lo amerite. Con lo cual "se podrá avanzar en el conocimiento sobre las condiciones necesarias, y de manera más amplia, del dinamismo del sistema social" escolar (Maculan, Jiménez y Castellanos, 2015, p. 16)

\section{Referencias}

Arias, E. (2018). Un acercamiento a lo radical de la convivencia. Cultura. Educación y Sociedad 9(1), 59-68.

Arrieta, M. (2016). Los masones en el mundo: Geopolítica masónica. La historia de los hijos de la Luz. Córdoba: Editorial Almuzara.

Bertussi, G. (2014). Los valores y la moral en la escuela. Educação \& Sociedade, 64-80.

Bonilla, M. y Jiménez, D. (2013). La actividad lúdica como estrategia para el desarrollo integral de los niños de la estancia infantil" Arcoiris" de la SEDESOL en el municipio de Ixtenco, Tlaxcala. [Doctoral dissertation, 291]. Tlaxcala, Apetatitlán.

Camargo, C. (2015). La investigación como estrategia pedagógica en la Guajira, desde una perspectiva de la inclusividad y diversidad como nuevo paradigma para el desarrollo de habilidades, destrezas y competencias. Educación y ciudad, (29), 149-162.

De la Concepción, A. (2015). Concepciones sobre participación de niñas, niños y adolescentes: $\mathrm{Su}$ importancia en la construcción de la convivencia escolar. Cultura Educación y Sociedad 6(2), 9-28.

Díaz, M. (2002). Convivencia escolar y prevención de la violencia. Página Web.

Escobar, G., Ocampo, G. y Romero, M. (2017). Taller lúdico: una estrategia para el reconocimiento del otro en el preescolar desde el goce, el juego y el movimiento.

García, P., Cantó, E., López, C., Miñarro, P. y Martínez, A. (2011). Análisis de la convivencia escolar en aulas de educación primaria. Revista Iberoamericana de Educación, 55(3), 1-12. 
García, L. y Niño, S. (2018). Percepciones sobre convivencia escolar y bullying en una institución educativa de Bogotá. Cultura, Educación y Sociedad 9(1), 4658.

García, M. (2003). El juego como estrategia didáctica para canalizar la agresividad infantil en preescolar. [Doctoral dissertation, UPN-162].

Guamani, G. y Alexandra, F. (2015). Programas de expresión corporal como estrategia lúdica para afianzar su esquema corporal en los párvulos de la escuela "Ramón Páez" del cantón Latacunga, provincia de Cotopaxi, sector Patután durante el periodo 2012-2013 [Bachelor's thesis, LATACUNGA/ $\mathrm{UTC} /$ ].

Hernández, H. y Jiménez, A. (2015). Responsabilidad social empresarial, zona urbana del distrito de Barranquilla: estudio etnográfico. Económicas CUC, 36(2), 9-18.

Herrera, K. y Rico, R. (2014). El clima escolar Como Elemento Fundamental de la Convivencia en la Escuela. Revista Escenarios. 12. 3-15.

Ledesma, C. (2012). Uso y Distribución de Espacios Escolares. Valencia: Escuela universitaria de educación de Valencia.

López, C., Carvajal, C., Soto, M. y Urrea, P. (2013). Factores asociados a la convivencia escolar en adolescentes. Educación y Educadores, 16(3). 383-410.

Lugo, L. (2007). Formación integral: desarrollo intelectual, emocional, social y ético de los estudiantes. Revista universitaria de sonora, (1). 1-3.

Maculan, A.-M., Jiménez , C. y Castellanos, O. (2015). Aprendizaje en el proceso de incubación de empresas de base tecnológica. Económicas CUC, 36(1), 1548.
Manilla, L. (2014). Estrategias para transformar el aula en espacio de convivencia pacífica. [Doctoral dissertation, 95].

Mariño, G. (2010). El diálogo en la educación de jóvenes y adultos. Dos propuestas pedagógicas para implementarlo. En: El taller dialógico / la recuperación de experiencias laborales. Bogotá. OEI.

Medina, A. y Quintero, J. (2003). El juego como estrategia didáctica para enriquecer el lenguaje en niños de edad preescolar. [Doctoral dissertation, UPN-25A].

Mejía, M. y Manjarrés, M. (2010). La Investigación como Estrategia Pedagógica. Programa Ondas-Colciencias.

Meléndez, N. y Juárez, A. (2012). Estrategias de enseñanza para aproximar a los niños a la adquisición del lenguaje escrito en 3er. año de preescolar. [Doctoral dissertation, UPN-Ajusco].

Mendoza, D. y López, D. (2015). Pensamiento estratégico: centro neurálgico de la planificación estratégica que transforma la visión en acción. Económicas CUC, 36(1), 153-179.

Mendoza, D., López, D. y Salas, E. (2016). Planificación estratégica de recursos humanos: efectiva forma de identificar necesidades de personal. Económicas CUC, 37(1), 61-79.

Ocampo, D. (2008). Los espacios urbanos recreativos como herramienta de productividad. Revista Escuela de Administración de Negocios, (63).

Ortega, P., Peñuela, D. y López, D. (2009). Sujetos y prácticas de la pedagogía crítica. Bogotá, D.C.: Ediciones El Búho.

Parra, J. (2005). La educación Infantil: su dimensión didáctica y organizativa. Granada: Grupo Editorial Universitario. 
Perales, C., Arias, E. y Bazdresch, M. (2014). Desarrollo socioafectivo y convivencia escolar. Tlaquepaque: ITESO.

Pozo, J. y Gómez, M. (2006). Aprender y enseñar ciencia. Madrid: Ediciones Morata.

Rivera, A. (2002). Estrategias para favorecer la expresión oral en el nivel preescolar del medio indigena. [Doctoral dissertation, UPN-31A].
Romero, A. (2015). El juego como estrategia para favorecer el logro de aprendizajes significativos de los niños y las niñas de 5 a 6 años de preescolar lll del jardín de niños Eretbim, de la Delegación Tlalpan del DF. [Doctoral dissertation, 99]. 